\title{
Reduced Synaptic Facilitation between Pyramidal Neurons in the Piriform Cortex After Odor Learning
}

\author{
Drorit Saar, ${ }^{1}$ Yoram Grossman, ${ }^{1}$ and Edi Barkai ${ }^{2}$ \\ Departments of ${ }^{1}$ Physiology and ${ }^{2}$ Morphology, Faculty of Health Sciences and Zlotowski Center for Neuroscience, Ben- \\ Gurion University of the Negev, Beer-Sheva 84105, Israel
}

Learning-related cellular modifications were studied in the rat piriform cortex after operand conditioning. Rats were trained to discriminate positive cues in pairs of odors. In one experimental paradigm, rats were trained to memorize 35-50 pairs of odors ("extensive training"). In another paradigm, training was continued only until rats acquired the rule of the task, usually after learning the first two pairs of odors ("short training"). "Pseudotrained" and "naive" rats served as controls. We have previously shown that "rule learning" of this task was accompanied by reduced spike afterhyperpolarization in pyramidal neurons in brain slices of the piriform cortex. In the present study, synaptic inputs to the same cells were examined. Pairs of electrical stimuli applied to the intrinsic fibers that interconnect layer II pyramidal neurons revealed significant reduction in pairedpulse facilitation (PPF) in this pathway even after short training. PPF in shortly trained rats was reduced to the same extent as

The idea that memory is manifested at the cellular level by enhancement of synaptic connections between simultaneously activated neurons was suggested half a century ago (Hebb, 1949) and has been widely accepted since then (for review, see Bliss and Collingridge, 1993; Hawkins et al., 1993). However, behaviorally induced synaptic changes have been demonstrated only lately, both in vivo (Ahissar et al., 1992; Wilson and McNaughton, 1994; Rioult-Pedotti et al., 1998) and in vitro (Mackernan and ShinnickGallagher, 1997; Power et al., 1997; Rioult-Pedotti et al., 1998). These last in vitro studies have demonstrated that learning-related synaptic modulations can be preserved and detected in brain slices.

The rat olfactory modality offers significant advantages for the study of learning-related synaptic modifications. Rats, for whom olfaction is a dominant sensory modality, can easily learn to discriminate between positive and negative cues in pairs of odors. Furthermore, rats demonstrate capability for rule learning of odor discrimination [e.g., interproblem learning that occurs when performing a series of discrimination problems with the same method of solution (Nirson et al., 1975)]. Thus, they can acquire large amount of olfactory information in discrimination tasks in a relatively short time (Saar et al., 1998). In addition, by applying "reversal test" (presenting previously learned odors with reversed significance), Staubli et al. (1987) demonstrated that rats can

\footnotetext{
Received March 1, 1999; revised July 14, 1999; accepted July 14, 1999.

This research was funded by a grant from the Israel Science Foundation.

Correspondence should be addressed to Dr. Edi Barkai, Department of Morphology, Faculty of Health Sciences, Ben-Gurion University of the Negev, Beer-Sheva 84105, Israel.

Copyright (C) 1999 Society for Neuroscience $\quad 0270-6474 / 99 / 198616-07 \$ 05.00 / 0$
}

in extensively trained rats. PPF reduction did not result from modification of membrane properties in the postsynaptic cells, change in postsynaptic inhibition, or impairment of the facilitation mechanism. Extracellular field potential recordings showed enhanced synaptic transmission in these synapses. The reduction in PPF became apparent only $3 \mathrm{~d}$ after task acquisition and returned to control value $5 \mathrm{~d}$ later. PPF evoked by stimulating the afferent fibers to the same neurons was increased $1 \mathrm{~d}$ after training for $2 \mathrm{~d}$. We suggest that the transient enhancement in connectivity in the intrinsic pathway is related to the enhanced learning capability and not to memory for specific odors, which lasts for weeks.

Key words: odor learning; operand conditioning; paired-pulse facilitation; piriform cortex; synaptic enhancement; pyramidal neurons

reliably recall previously learned odors few weeks after training, even if additional odor memory was acquired during that time.

The piriform cortex is the largest cortical area receiving direct input from the olfactory bulb, via the lateral olfactory tract (LOT), without thalamic intermediation. The inputs of the olfactory nerve are nontopographically spread across the entire surface of the piriform cortex. Accordingly, presentation of eight different odors to rats resulted in increased firing rate in $>30 \%$ of the piriform cortex cells, with each cell responding to at least one of the odors (Schoenbaum and Eichenbaum, 1995a).

Activity-dependent plasticity in the piriform cortex has been reported in several studies. Synaptic activity evoked in the piriform cortex by stimulating the LOT is strongly enhanced by olfactory training (Roman et al., 1987, 1993; Litaudon et al., 1997). LTP can be readily induced in the piriform cortex in vitro (Jung et al., 1990; Kanter and Haberly, 1990, 1993; Jung and Larson 1994; Hasselmo and Barkai, 1995) and in vivo (Stripling et al., 1988, 1991). The piriform cortex has a simple and defined anatomical organization (Price, 1973; see also Fig. 1C). Pyramidal cell bodies are densely packed in a thin layer (layer II), with the intercortical association axons synapsing on the proximal zone of the apical dendrites (layer Ib), and the afferent input axons of the LOT synapsing on the distal part of the apical dendrites (layer Ia). This laminar organization enables recording from a homogenous population of neurons and stimulating specific synaptic pathways.

We have previously demonstrated that rule learning of an odor discrimination task is accompanied by increased excitability in pyramidal neurons in layer II of the piriform cortex, caused by reduced spike afterhyperpolarization (Saar et al., 1998). We 

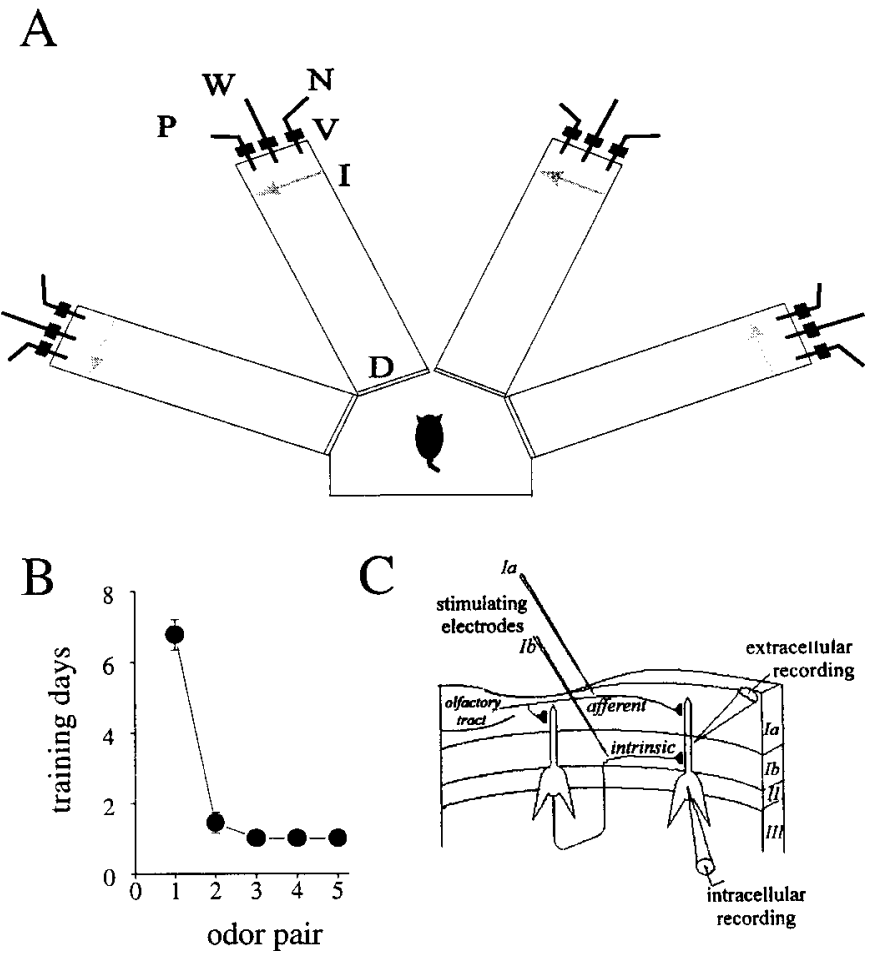

Figure 1. Training for odor discrimination and recording from cortical brain slices. $A$, Schematic description of the four-arm maze. Protocols for trained and pseudotrained rats were similar: an electronic "start" command opens randomly two of eight valves $(V)$, releasing a positive-cue odor $(P)$ into one of the arms and a negative-cue odor $(N)$ into another. Eight seconds later, the two corresponding guillotine doors $(D)$ are lifted to allow the rat to enter the selected arms. After reaching the far end of an arm (90-cm-long), the rat body interrupts an infrared beam (I, arrow), and a drop of drinking water is released from a water hose $(W)$ into a small drinking well (for a trained rat, only if the arm contains the positive-cue odor; for a pseudotrained rat, randomly). A trial ends when the rat interrupts a beam, or in $10 \mathrm{sec}$, if no beam is interrupted. A fan is operated for $15 \mathrm{sec}$ between trials, to remove odors. $B$, Trained rats demonstrated acquisition of rule learning. Seven consecutive days of training were required for this group to reach criterion for discriminating between the first pair of odors ( $80 \%$ correct choices). Discrimination between any new pair of odors, starting from the third and fourth pairs could be reached within 1 d. Values represent mean \pm SE. $n=11$ rats. Results were similar for other rat groups that were trained subsequently. $C$, Schematic illustration of the piriform slice and the experimental procedure. The pyramidal cells located in layer II receive excitatory afferent input from LOT at the distal dendrites (layer $I a$ ) and excitatory intrinsic input from other cortical pyramidal cells at the proximal dendrites (layer $I b$ ). Intracellular recordings were performed from cell bodies in layer II. Field potentials were recorded in layer $I b$.

report now that synaptic transmission between these neurons is also enhanced after acquisition of the same task. Furthermore, we show that this pathway-specific synaptic enhancement is transient, and thus cannot underlie long-term memory storage.

\section{MATERIALS AND METHODS}

\section{Animal training}

Subjects. Young adult Sprague Dawley male rats were used. Before training they were maintained on a $23.5 \mathrm{hr}$ water deprivation schedule, with food available ad libitum.

Apparatus and odors. Olfactory discrimination training protocol was performed in a four-arm radial maze (Fig. $1 A$ ), with commercial odors that are regularly used in the cosmetics and food industry.

Training. Olfactory training consisted of 20 trials per day for each rat. Learning was considered as acquired after demonstration of at least $80 \%$ positive cue choices in the last 10 trials of the day. The control rats were either exposed to the same protocol of training, but with random water rewarding (pseudotrained) or were water-deprived, with no training (naive). Once discrimination between a pair of odors was acquired by all the rats in the trained group, on the next day both trained and pseudotrained groups resumed training with a new pair of unfamiliar odors. As we previously reported (Saar et al., 1998), our training study confirms the original report by Staubli et al. (1987) that once the rats reach good performance with the first pair of odors, their capability to distinguish between new odors increased (Fig. $1 B$ ). These data suggest an important implication: training to distinguish between the first pair of odors results not only in memory acquisition of these particular odors, but with the ability to acquire odor memory much faster. Therefore, one may speculate that rule learning was also acquired. According to the experiment, some rats were trained with two or three pairs of odors, to ensure rule learning (short training). Others were trained with up to 50 pairs of odors (extensive training).

\section{Slice preparation, stimulation, and recording}

Rats were killed at several different time periods after training completion. Four hundred micrometer coronal brain slices were cut as previously described (Barkai and Hasselmo, 1994) and kept in oxygenated $\left(95 \% \mathrm{O}_{2}\right.$ plus $5 \% \mathrm{CO}_{2}$ ) Ringer's solution (in mM: $\mathrm{NaCl} 124, \mathrm{KCl} 3$, $\mathrm{MgSO}_{4} 2, \mathrm{NaH}_{2} \mathrm{PO}_{4} 1.25, \mathrm{NaHCO}_{3} 26, \mathrm{CaCl}_{2} 2$, and glucose 10). Tungsten electrodes were placed in layer $\mathrm{Ib}$ to stimulate the intrinsic fibers and in layer Ia to stimulate the afferent fibers (Fig. 1C). Electrical stimuli were applied at $0.1 \mathrm{~Hz}$. Intracellular recordings were performed at $36^{\circ} \mathrm{C}$ with $4 \mathrm{M} \mathrm{K}$-acetate-filled sharp electrodes. The amplitudes of the responses were measured from digital averaging of 10 consecutive responses. To standardize the intracellular recording conditions, stimulus intensity was adjusted so that the averaged amplitude of 10 consecutive PSPs in the recorded cell would be $10 \mathrm{mV}$ at $V \mathrm{~m}=-80 \mathrm{mV}$, and the same stimulus intensity was used for all paired pulse facilitation (PPF) measurements in that cell. Extracellular recordings were performed with Ringer's solution-filled electrodes at lower temperature $\left(34^{\circ} \mathrm{C}\right)$, to allow temporal separation between the presynaptic and postsynaptic responses.

The identity of rats (naive, trained, or pseudotrained) was not known to the person conducting the experiments and the analysis.

\section{Statistical analysis}

One-way ANOVA was used to evaluate significance of difference among three cell populations. Student's $t$ test was used to compare between each two cell populations.

\section{RESULTS}

\section{Extensive training}

The kinetics of single PSPs in the intrinsic synapses are not modified by training

We have previously shown that basic membrane properties such as resting potential, input resistance, and membrane time constant in layer II pyramidal neurons are not modified after extensive training (Saar et al., 1998). The kinetics of single, standardized $(10 \mathrm{mV}$ amplitude at $V \mathrm{~m}=-80 \mathrm{mV})$ postsynaptic potentials, evoked by stimulating the intrinsic pathway to these neurons, were also similar in all groups (Table 1). These data suggest that the learning-related modifications in the dynamics of these synaptic responses, as will be described, are not the result of changes in membrane properties of the postsynaptic neurons.

\section{$P P F$ in the intrinsic synapses is decreased after training}

Pairs of stimuli, separated by short intervals, may result in amplification of the second response in a pair. This phenomenon, termed PPF, is thought to reflect enhanced synaptic release during the second response, caused by residual $\mathrm{Ca}^{2+}$ accumulation in the presynaptic terminal (Katz and Miledi, 1968; Wu and Saggau, 1994). Pyramidal cells in the piriform cortex exhibited PPF when interstimulus intervals (ISI) ranged between 50 and $150 \mathrm{msec}$. For this whole range, PPF was markedly smaller in cells from the trained rats than in cells from pseudotrained and naive rats (Fig. 2A,B). The SD of PPF distribution for the trained group 
Table 1. Single EPSP kinetics in neurons from the three groups of rats after extensive training

\begin{tabular}{|c|c|c|c|}
\hline & $\begin{array}{l}\text { Rate of rise } \\
(\mathrm{mV} / \mathrm{msec})\end{array}$ & $\begin{array}{l}\tau \text { of decay } \\
(\mathrm{msec})\end{array}$ & $\begin{array}{l}\text { Half-width } \\
\text { (msec) }\end{array}$ \\
\hline Naive & $\begin{aligned} 1.46 & \pm 0.79 \\
n & =18\end{aligned}$ & $\begin{aligned} 35.2 & \pm 16.2 \\
n & =19\end{aligned}$ & $\begin{aligned} 30.9 & \pm 14.8 \\
n & =17\end{aligned}$ \\
\hline Trained & $\begin{array}{c}1.39 \pm 0.54 \\
n=16\end{array}$ & $\begin{aligned} 35.7 & \pm 12.2 \\
n & =16\end{aligned}$ & $\begin{aligned} 27.3 & \pm 5.7 \\
n & =16\end{aligned}$ \\
\hline Pseudotrained & $\begin{aligned} 1.31 & \pm 0.50 \\
n & =9\end{aligned}$ & $\begin{aligned} 37.3 & \pm 20.9 \\
n & =11\end{aligned}$ & $\begin{aligned} 29.1 & \pm 9.6 \\
n & =9\end{aligned}$ \\
\hline
\end{tabular}

Stimuli were applied at $0.1 \mathrm{~Hz}$. Stimulus intensity was adjusted to evoke EPSP with amplitude of $10 \mathrm{mV}$ at membrane potential of $-80 \mathrm{mV}$, and the responses in 10 consecutive trials were digitally averaged. EPSP rate of rise was measured from onset to peak. EPSP $\tau$ of decay was measured by fitting a single exponential decay curve. EPSP half-width is the time interval between points at the level of 0.5 of the EPSP amplitude. Each value represents mean \pm SD. Each group consisted of seven rats; $n=$ number of neurons.

was not larger than for the other groups. For example, at ISI of 50 msec the values of PPF were $1.33 \pm 0.21, n=20$ for the naive, $1.19 \pm 0.17, n=20$ for the trained, and $1.37 \pm 0.24, n=13$ for the pseudotrained group. This indicates that PPF reduction was apparent in most of the neurons sampled in trained rats, rather than in a subgroup of these neurons, as demonstrated in the cumulative frequency distribution of PPF values (Fig. 3A). Figure $3 B$ demonstrates that PPF reduction became prominent $1 \mathrm{~d}$ after extensive training completion and remained so until $6 \mathrm{~d}$ after the training. Dysfunction of the PPF mechanism after intense training was ruled out by perfusing the slices from trained rats with low $\left[\mathrm{Ca}^{2+}\right](0.5 \mathrm{~mm})$ solution, as a result of which the first EPSP was reduced, and PPF was enhanced ( $n=3$; Fig. $4 A$ ).

\section{PPF in the intrinsic synapses is not reduced by increased} postsynaptic conductance

EPSPs evoked by stimulation of the intrinsic fibers in the piriform cortex are usually followed by a fast, $\mathrm{GABA}_{\mathrm{A}}$-mediated IPSP, and a late, $\mathrm{GABA}_{\mathrm{B}}$-mediated IPSP, which is at its peak $150 \mathrm{msec}$ after stimulation (Tseng and Haberly, 1988). Indeed, the decay rate of the second EPSP was always faster compared to that of the first EPSP, indicating increased membrane conductance in the postsynaptic cell during the second EPSP. This raises the possibility that a slow postsynaptic conductance, initiated by the first stimulus of the pair, underlies an apparent PPF reduction. However, the ratio between decay rate of the second and the first EPSPs was similar for all groups (Fig. 4B), suggesting that the shunt caused by evoked postsynaptic conductance was similar for all groups and could not underlie the difference in PPF between the trained and the control rats.

\section{Short training}

PPF reduction in the intrinsic synapses is apparent also after short training

The next set of experiments was aimed to determine whether PPF reduction in the piriform cortex is correlated to the massive storage of memory of many odors. To address this question, PPF was examined in neurons from rats that were trained with only two pairs of odors, until the dramatic enhancement in their capability to learn new odors was demonstrated (Fig. 1B; Staubli et al., 1987; Saar et al., 1998), but only few new odors are stored in memory. PPF with ISI $=50 \mathrm{msec}$ was compared among these shortly trained rats, shortly pseudotrained, and naive rats. Averaged PPF values in the shortly pseudotrained (mean $\pm \mathrm{SE}, 1.33 \pm$
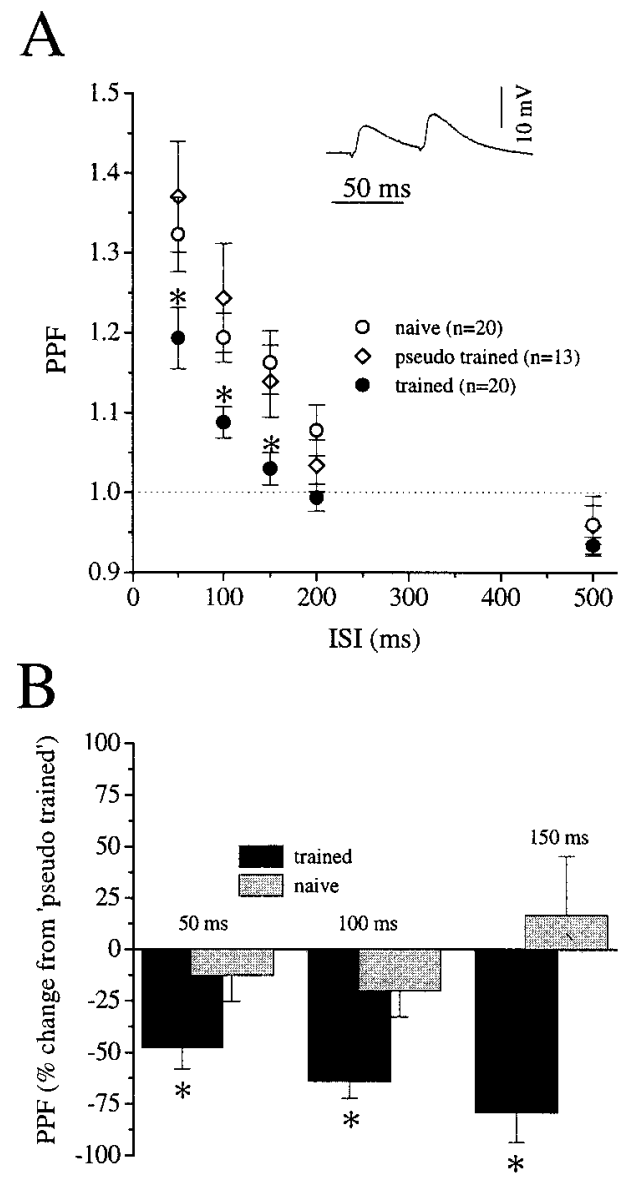

Figure 2. Reduced PPF in piriform cortex of extensively trained rats. Each group entailed seven rats. Rats were killed 1-6 d after training completion. $A$, PPF was maximal at ISI $=50 \mathrm{msec}$ and decreased with larger intervals. For each ISI between 50 and $150 \mathrm{msec}$, the averaged PPF measured in neurons from trained rats was significantly smaller compared to that in neurons from naive and pseudotrained $\left({ }^{*} p<0.04\right)$ rats. PPF was determined by calculating the ratio between the amplitude of the second and first PSPs $\left(\mathrm{PSP}_{2} / \mathrm{PSP}_{1}\right)$ in digitally averaged 10 consecutive responses. When the second PSP overlapped the late part of the first, its baseline was estimated by digitally subtracting a trace of averaged 10 single PSPs evoked in the same cell. Each value represents mean \pm SE. $n=$ number of neurons. One to four neurons were recorded form each animal in the trained and naive groups, and one to three neurons were recorded from each animal in the pseudotrained group. Inset, A typical average of 10 responses to pairs of stimuli applied at $0.1 \mathrm{~Hz}$ with ISI $=$ $50 \mathrm{msec}$. $B$, PPF in trained and naive rats (same neurons as in $A$ ) presented as percent of PPF in neurons from the pseudotrained group $\left(\left(\mathrm{PPF}-1 / \mathrm{PPF}_{\mathrm{pseudo}}-1\right) * 100\right)$. PPF in trained rats was reduced to $50-75 \%$ of PPF value in the pseudotrained rats $\left({ }^{*} p<0.04\right)$, whereas PPF in naive rats did not significantly differ from the pseudotrained. Values represent mean $\pm \mathrm{SE}$.

$0.15 ; n=38$ cells $)$ and in the naive $(1.28 \pm 0.22 ; n=23)$ groups were similar to each other and also similar to the PPF values in extensively pseudotrained $(1.37 \pm 0.25 ; n=13)$ and their matching naive $(1.32 \pm 0.12 ; n=20)$ groups. PPF in the shortly trained group reached a minimum value that was similar to the decrease previously observed in extensively trained rats. Also, $8 \mathrm{~d}$ after training completion PPF returned to control value (Fig. $5 A$ ). The values of PPF in neurons from trained rats at different time periods after training were as follows: $1 \mathrm{~d}, 1.31 \pm 0.14 ; 2 \mathrm{~d}, 1.31 \pm$ $0.19 ; 3 \mathrm{~d}, 1.17 \pm 0.14 ; 5 \mathrm{~d}, 1.11 \pm 0.02 ; 7 \mathrm{~d}, 1.09 \pm 0.05 ; 8 \mathrm{~d}, 1.30 \pm$ 0.16 ; and $10 \mathrm{~d}, 1.32 \pm 0.12$.

Cumulative frequency histograms show that here too, the PPF 
A

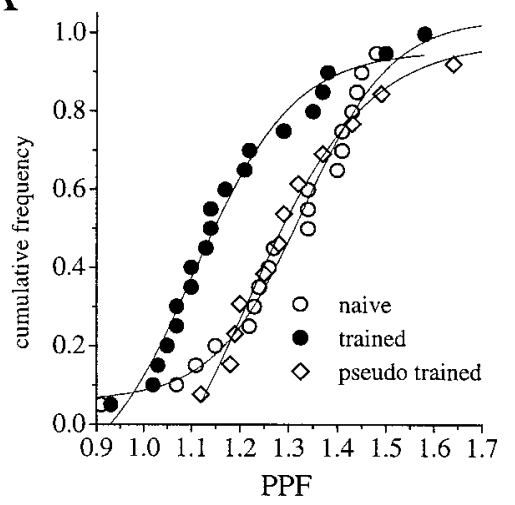

$\mathrm{B}$

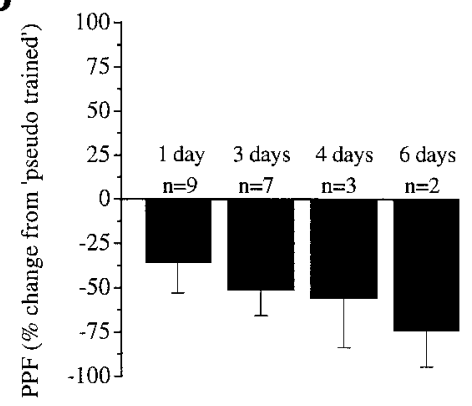

Figure 3. PPF reduction after extensive training is apparent in most neurons. $A$, Cumulative frequency distribution of PPF values at ISI $=50$ msec (each point represents PPF in one cell, the same cells as in Fig. $2 A$ ). The curve of PPF values in trained rats is smoothly shifted to the left, indicating that PPF reduction occurred in most of the sampled neurons and not in a subgroup. $B$, Dynamics of PPF decrease (ISI $=50 \mathrm{msec}$ ). PPF in neurons from the trained rats (same neurons as in Fig. $2 A$ ) presented as percent of the averaged PPF in neurons from the pseudotrained rats, and grouped according to the time periods after training completion: three rats after $1 \mathrm{~d}$, two rats after $3 \mathrm{~d}$, one rat after $4 \mathrm{~d}$, and one rat after $6 \mathrm{~d}$. PPF reduction was prominent $1 \mathrm{~d}$ after training completion and remained so until $6 \mathrm{~d}$ after the training. The apparent gradual decrease from day 1 to day 6 was not significant, and thus the results were lumped together in the curve in $A$. Values represent mean \pm SE. $n=$ number of cells.

reduction was detected in most of the sampled neurons and not in a subgroup (Fig. 6).

\section{Synaptic responses, but not axonal excitability is enhanced in} the intrinsic fibers

With short electrical stimulation applied to the intrinsic axons, extracellular recordings in layer II reveal field potentials with two separable components: a fast, short component, reflecting action potentials in the activated axons (axon volley) and a slower component, which could be abolished by $\mathrm{Ca}^{2+}$ removal, representing the PSPs generated in the pyramidal neurons (Fig. 7A, top). As stimulus intensity was increased, a linear increase was observed in both components (Fig. $7 A$, bottom, $B$ ). The ratio between stimulus intensity and the evoked axon volley did not differ between trained and the control groups (Fig. 7C), indicating that training did not alter the intrinsic axonal excitability in the cortex. However, the ratio between field postsynaptic potential (fPSP) amplitude and axon volley increased significantly after training (Fig. 7D), indicating that the synaptic transmission was enhanced.
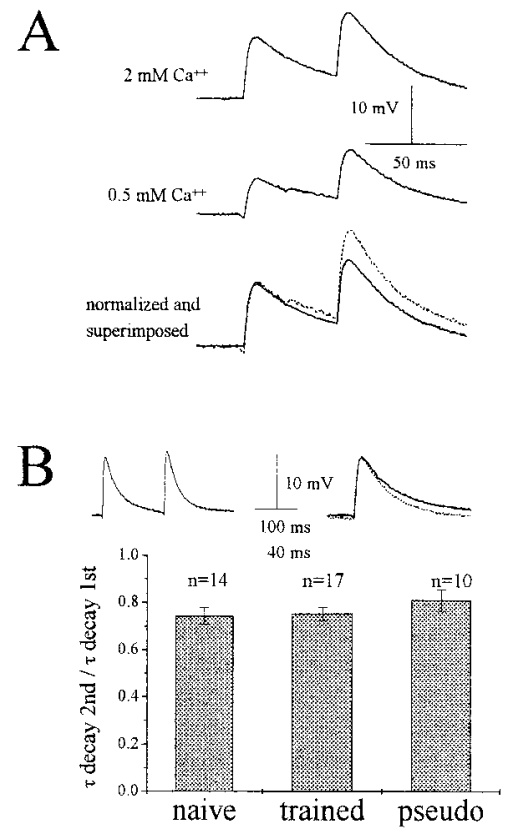

Figure 4. Properties of PPF reduction. $A$, Effect of low $\left[\mathrm{Ca}^{2+}\right]_{\mathrm{o}}$ on PPF in neuron from trained rat. The first response of the neuron to a pair of stimuli in normal $\left[\mathrm{Ca}^{2+}\right]_{\mathrm{o}}(2 \mathrm{mM}$, top trace $)$ was reduced when the neuron was perfused with low $\left[\mathrm{Ca}^{2+}\right]_{\mathrm{o}}$ solution $(0.5 \mathrm{mM}$, middle trace). Superimposition of the two traces after normalizing the amplitudes of the first PSPs in both (bottom) reveals that PPF was increased. $B$, Evoked postsynaptic late inhibitory conductance does not change after training. Top, The left trace demonstrates a typical response to paired stimuli, ISI $=150$ msec. Superimposition (right) reveals that the second response (dots) decays faster than the first (line), suggesting that the first stimulus has evoked a late postsynaptic shunting conductance. Bar graph, The ratio between decay time constants of the second and the first PSPs did not differ between groups, suggesting that the late postsynaptic conductance did not change after training. Values represent mean \pm SE. $n=$ number of cells.

\section{PPF increase in synapses of the afferent fibers}

The profound enhancement in synaptic transmission after short training raises the question whether it is a general arousal phenomenon in the piriform cortex. Therefore, we examined the PPF of synaptic responses evoked by stimulating the afferent axons terminating on the same layer II pyramidal neurons. The synaptic potentials evoked by stimulating at layer Ia, which contains these afferent fibers (Price, 1973), had PPF value of $1.21 \pm 0.13(n=$ $23)$ in slices from pseudotrained and $1.22 \pm 0.10(n=11)$ in naive rats. In contrast to the effect of training on PPF in the intrinsic pathway, PPF in the afferent pathway did not decrease after rule learning acquisition. Rather, 1 to $2 \mathrm{~d}$ after training completion, PPF in the afferent pathway increased, and $3 \mathrm{~d}$ after training completion PPF in this pathway returned to control value (Fig. $5 B)$. The values of PPF in neurons from trained rats at different time periods after training were as follows: $1 \mathrm{~d}, 1.37 \pm 0.09 ; 2 \mathrm{~d}$, $1.36 \pm 0.10 ; 3 \mathrm{~d}, 1.19 \pm 0.13 ; 7 \mathrm{~d}, 1.18 \pm 0.06 ; 8 \mathrm{~d}, 1.19 \pm 0.10$; $10 \mathrm{~d}, 1.29 \pm 0.07$.

\section{DISCUSSION}

Our data show that synaptic transmission between layer II pyramidal neurons in the piriform cortex is altered after odor learning. Although the basic kinetics of the single PSPs did not change even after extensive training, in consistence with our previous finding that no changes occur in the intrinsic membrane properties of these neurons (Saar et al., 1998), PPF evoked by stimulat- 

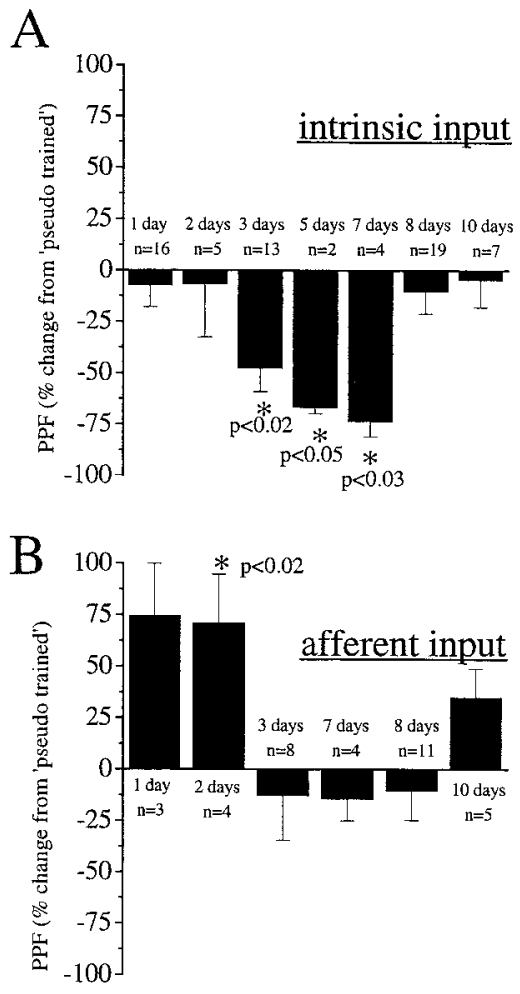

Figure 5. PPF is reduced in the intrinsic, but not afferent synaptic pathway after short training. Data obtained from 20 trained and 14 pseudotrained rats. $A$, Dynamics of PPF decrease in the intrinsic pathway after short training (stimuli applied to layer Ib; ISI $=50 \mathrm{msec}$ ). Values of PPF in shortly trained rats are presented as percent of the mean value of PPF in their matched shortly pseudotrained rats. Trained rats were killed at different time periods after training completion: four rats after $1 \mathrm{~d}$, two rats after $2 \mathrm{~d}$, four rats after $3 \mathrm{~d}$, one rat after $5 \mathrm{~d}$, two rats after $7 \mathrm{~d}$, five rats after $8 \mathrm{~d}$, and two rats after $10 \mathrm{~d}$. Two to six neurons were recorded from each animal in the trained, pseudotrained, and naive groups. Values represent mean \pm SE. $n=$ number of cells. $B$, Dynamics of PPF increase in the afferent pathway after short training. The same cells as in $A$, with stimuli applied to layer Ia, ISI $=50 \mathrm{msec}$. Values of PPF in the afferent pathway in trained rats described as percent of the mean value in the same pathway in pseudotrained rats. Values represent mean \pm SE. $n=$ number of neurons.

ing the intrinsic fibers was reduced after short odor training. In addition, the ratio between field PSP and axon volley in the same pathway was increased after training. Our findings suggest that synaptic transmission in the intrinsic pathways is temporarily increased for several days after short training.

\section{The nature of enhanced synaptic connectivity}

It has been shown previously that odor discrimination learning is accompanied by potentiation in the piriform cortex (Roman et al., 1987, 1993) and that such modifications are spread over large areas of the piriform cortex (Litaudon et al., 1997). Our data support these findings. Gradual increase in the number of activated axons in the intrinsic pathway resulted with significantly greater increase of the fPSP in "trained" rats, compared to both controls, indicating enhanced synaptic transmission. At the single cell level, PPF was markedly reduced in neurons from trained rats, and this reduction was not the result of increase in evoked postsynaptic conductance. Both results indicate that the synaptic transmission enhancement is, at least in part, the result of augmented synaptic release. i.e., the release caused by the first stimulus in a pair is closer to its maximal value, leaving less room

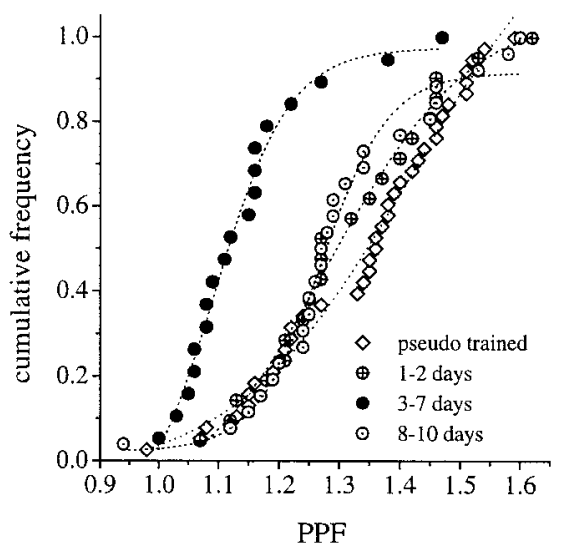

Figure 6. PPF reduction in the intrinsic pathway after short training is observed in most sampled neurons. Cumulative frequency distribution of PPF values at ISI $=50 \mathrm{msec}$. Each point represents PPF in one cell, the same cells as in Figure $5 A$. PPF values between 3 and $7 \mathrm{~d}$ did not differ significantly and therefore are presented together. The curve of PPF values $3-7 \mathrm{~d}$ after training completion is smoothly shifted to the left, indicating that PPF reduction occurred in most of the sampled neurons and not in a subgroup. The curve of PPF values in the first $2 \mathrm{~d}$, and $8 \mathrm{~d}$ or more after training completion did not differ from the curve of the pseudotrained rats.

for further facilitation of the second response (Debanne et al., 1996). Indeed, when $\left[\mathrm{Ca}^{2+}\right]_{0}$ was reduced to decrease the release, PPF in slices from trained rats increased.

\section{Specificity of modifications in synaptic transmission to learning}

The physiological changes observed in the cortex of the trained animals were detected neither in the pseudotrained rats, which were exposed to the same odors, nor in naive rats. This suggests that exposure to odors or to the four-arm maze in itself is not sufficient to generate the observed synaptic modifications (Saar et al., 1998), rather they are correlated with the learning process. Furthermore, only recent odor learning will be reflected in PPF reduction, because $8 \mathrm{~d}$ after training the effect disappears. Enhanced synaptic transmission has been shown lately in brain slices from amygdala after fear conditioning (Mackernan and ShinnickGallagher, 1997) and in hippocampus after eyeblink conditioning (Power et al., 1997), suggesting that learning-related enhancement in synaptic transmission may be a general phenomenon that takes place in task-relevant regions of the mammalian brain after different types of learning. Furthermore, the reduction of PPF in the piriform cortex was specific to the intrinsic pathway, suggesting that, as in the amygdala (Mackernan and Shinnick-Gallagher, 1997), enhanced synaptic transmission in the piriform cortex occurs at specific relevant sites and not throughout the local cortical circuit.

That the reduced PPF occurs after extensive training to the same extent as after short training may indicate that this modification reflects the existence of enhanced learning capability. Alternatively, it may reflect an ability to devote special attention to odor stimuli, which becomes crucial for the trained rats.

\section{Slow onset to maximal PPF reduction}

The finding that PPF reduction after short training appears only $3 \mathrm{~d}$ after the last training session is of particular interest. One possible explanation for this slow onset is that it reflects decreased responsiveness in the olfactory system, which occurs as a result of intense activity during odor learning, and fades out 

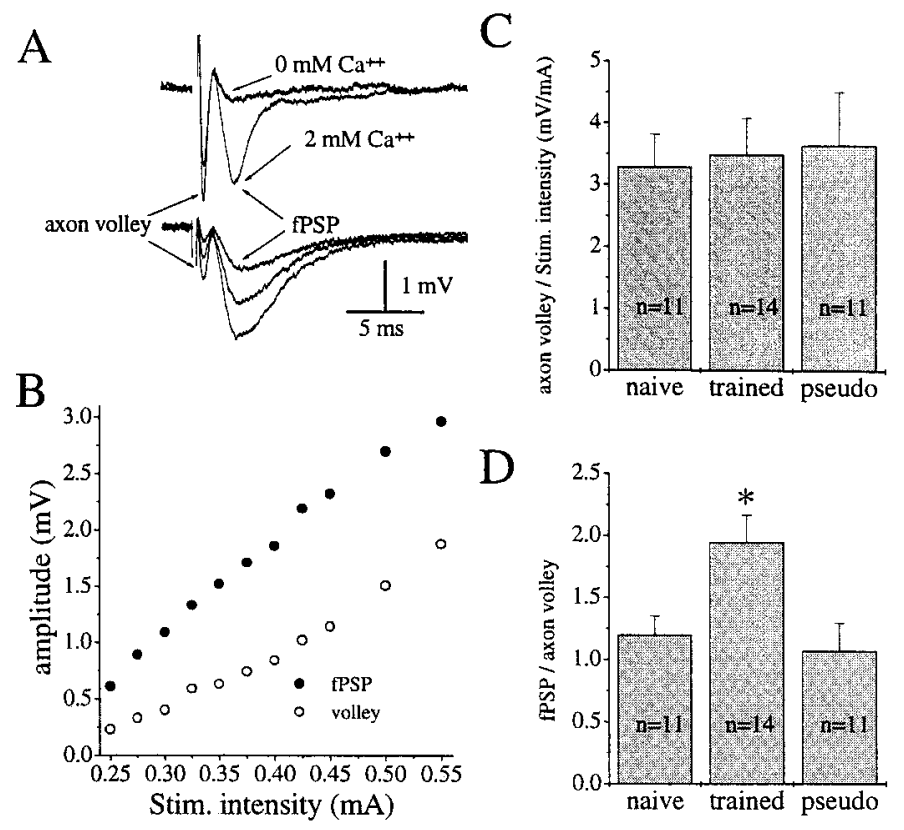

Figure 7. Enhanced synaptic response in the intrinsic pathway in shortly trained rats. Rats were killed 3-6 d after training completion. $A$, Extracellular field potentials in response to short stimulations of the intrinsic fibers. The axon volley component is followed by a postsynaptic component ( $f P S P)$. Top, Removal of $\mathrm{Ca}^{2+}$ from the perfusing Ringer's solution $\left(2 \mathrm{mM} \mathrm{CaCl}_{2}\right.$ was replaced by $2 \mathrm{mM} \mathrm{MgCl}_{2}$ ) resulted with increase of the axon volley and complete abolishment of the fPSP. Bottom, In normal Ringer's solution both components increase with increasing stimulus intensity (up to $0.55 \mathrm{~mA}$ in this case). $B$, Example of axon volley and fPSP amplitudes in response to different stimulus intensities. Both components of the field potential show linear relation to stimulus intensity. $C$, Averaged values of the slopes of axon volley amplitude versus stimulus intensity. Slopes were calculated by applying linear fits to the graphs as in $B$. $n=$ number of slices. $D$, Averaged values of the slopes of fPSP amplitudes versus axon volley amplitudes. Slopes were calculated by applying linear fits to the graphs. The same slices as in $C$. Note the significant difference between the trained group and the pseudotrained and naive groups $\left({ }^{*} p<\right.$ $0.05)$. Each group entailed seven rats. One or two field potentials were recorded from each animal.

within $3 \mathrm{~d}$ after the training. Such decreased responsiveness, manifested at the synaptic level as reduced synaptic transmission, is consistent with the transient increase in PPF in the afferent synaptic connections, which appears $1 \mathrm{~d}$ after training and lasts for $2 \mathrm{~d}$. The notion that PPF increase in the afferent pathway reflects a temporary reduction in synaptic transmission suggests that the afferent synapses are not enhanced by training. However, if both processes occur in parallel in the intrinsic pathway, the effect of synaptic potentiation on PPF would be recorded only after the antagonist process is diminished.

\section{Behavioral relevance of PPF reduction}

We suggest that the observed PPF reduction in the intrinsic pathway in the piriform cortex of odor-trained rats is related to the phenomenon of enhanced learning rate after rule learning acquisition rather than to memory storage of specific odors for the following reasons: (1) PPF is significantly decreased after short training, which results with rule learning acquisition, to the same extent as it is decreased after memorizing many odors after extensive training (Fig. 8A), and was apparent in most of the sampled neurons. Such a whole network modification is suitable to serve increased information processing capability. It is unlikely, though, that memory storage of two or many tens of odors
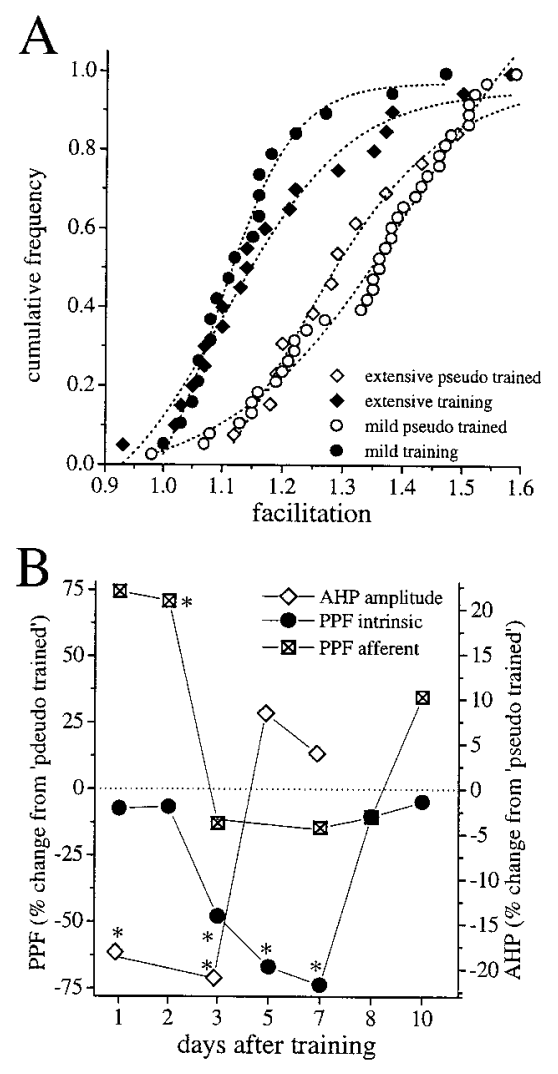

Figure 8. A, Cumulative frequency distribution of PPF in shortly trained and extensively trained rats and their matching pseudotrained groups (same data as in Figs. $3 A$ and 6). Distribution of both trained groups are similar, as is the distributions of both pseudotrained groups. B, Time course of AHP reduction (Saar et al., 1998) and PPF changes in the intrinsic and afferent pathways after short training completion. Values are normalized to the averaged corresponding values in pseudotrained rats.

will be represented by exactly the same quantitative decrease in PPF. (2) Training suspension for $8 \mathrm{~d}$ or more results with PPF return to pretraining values. We have previously shown that such a break in the daily training routine results with reduced capability to learn new odors (Saar et al., 1998). In contrast, rats can retrieve memory for learned odors for at least 6 weeks after learning (Staubli et al., 1987).

The question regarding the final location of long-term odor memory storage remains open. One possibility is that long-term modifications underlying memory also occur in the piriform cortex, but that synaptic strengthening is mediated by increased number of synapses rather than by physiological changes at single synapses. Another possibility is that long-term memory modifications occur at a different brain area, such as the orbitofrontal cortex, which is very active in odor learning (Schoenbaum and Eichenbaum 1995a,b; Eichenbaum et al., 1996; Schoenbaum et al., 1999).

\section{Functional significance of the time window in which PPF is reduced}

Learning-dependent cellular modifications that lag the last training session by $3 \mathrm{~d}$ were shown in dendrites of hippocampal pyramidal neurons after classical conditioning (Olds et al., 1989). Recently we have shown that short training results with increased excitability in pyramidal neurons in layer II of the piriform cortex, caused by reduced spike afterhyperpolarization that can be detected $1 \mathrm{~d}$ after training and lasts for up to $3 \mathrm{~d}$ (Saar et al., 1998). 
Taken together with the present results, it appears that these two learning-related modifications, which emerge in sequence, with partial overlap on the third day after training, can maintain learning-related single-cell modifications for the combined period of $8 \mathrm{~d}$. Accordingly, we suggest the following model for enhanced learning capability in the mammalian brain: increased neuronal excitability enhances neuronal activation, which consequently, according to Hebb's rules, enhances synaptic transmission in the intrinsic fibers, as reflected in reduced PPF (Fig. 8B). This transient synaptic enhancement does not underlie long-term memory maintenance, but may serve as an intermediate stage, creating favorable conditions for long-term memory formation.

\section{REFERENCES}

Ahissar E, Vaadia E, Ahissar M, Bergman H, Arieli A, Abeles M (1992) Dependence of cortical plasticity on correlated activity of single neurons and on behavioral context. Science 257:1412-1415.

Barkai E, Hasselmo ME (1994) Modulation of the input/output function of rat piriform cortex pyramidal cells. J Neurophysiol 72:659-677.

Bliss TV, Collingridge GL (1993) A synaptic model of memory: long term potentiation in the hippocampus. Nature 361:31-39.

Debanne DN, Guerineau C, Gahweiler BH, Thompson SM (1996) Paired-pulse facilitation and depression at unitary synapses in rat hippocampus: quantal fluctuations affects subsequent release. J Physiol (Lond) 491:163-170.

Eichenbaum H, Schoenbaum G, Young B, Bunsey M (1996) Functional organization of the hippocampal memory system. Proc Natl Acad Sci USA 93:13500-13507.

Hasselmo ME, Barkai E (1995) Cholinergic modulation of activitydependent synaptic plasticity in the piriform cortex and associative memory function in a network biophysical simulation. J Neurosci 15:6592-6604.

Hawkins RD, Kandel ER, Siegelbaum SA (1993) Learning to modulate transmitter release: themes and variations in synaptic plasticity. Annu Rev Neurosci 16:625-665.

Hebb D (1949) The organization of behavior. New York: Wiley.

Jung MW, Larson J (1994) Further characteristics of long-term potentiation in piriform cortex. Synapse 18:298-306.

Jung MW, Larson J, Lynch G (1990) Long-term potentiation of monosynaptic EPSPs in rat piriform cortex in vitro. Synapse 6:279-283.

Kanter ED, Haberly LB (1990) NMDA-dependent induction of longterm potentiation in afferent and association fiber systems of piriform cortex in vitro. Brain Res 525:175-179.

Kanter ED, Haberly LB (1993) Associative long-term potentiation in cortex slices requires GABA blockade. J Neurosci 13:2477-2482.

Katz B, Miledi R (1968) The role of calcium in neuromuscular facilitation. J Physiol (Lond) 195:481-492.

Litaudon P, Mouly A-M, Sullivan R, Gervais R, Cattarelli M (1997)
Learning-induced changes in rat piriform cortex activity mapped using multisite recording with voltage-sensitive dye. Eur J Neurosci 9:1593-1602.

Mackernan MG, Shinnick-Gallagher P (1997) Fear conditioning induces a lasting potentiation of synaptic currents in vitro. Nature 390:607-610.

Nirson BJ, Slotnick BM, Nevin JA (1975) Olfactory discrimination, reversal learning, and stimulus control in rats. J Comp Physiol Psychol 89:285-294.

Olds JL, Matthew L, McPhie DL, Staten LD, Alkon DL (1989) Imaging of memory-specific changes in the distribution of protein kinase $\mathrm{C}$ in the Hippocampus. Science 245:866-869.

Power JM, Thompson LT, Moyer JR, Disterhoft JF (1997) Enhanced synaptic transmission in CA1 hippocampus after eyeblink conditioning. J Neurophysiol 78:1185-1187.

Price TH (1973) An autoradiographic study of complementary laminar patterns of termination of afferent fibers to the olfactory cortex. J Comp Neurol 150:87-108.

Rioult-Pedotti MS, Friedman D, Roman F, Staubli U, Donaghue JP (1998) Skill learning occludes LTP in horizontal cortical connections. Soc Neurosci Abstr 24:602.9.

Roman F, Staubli U, Lynch G (1987) Evidence for synaptic potentiation in a cortical network during learning. Brain Res 418:221-226.

Roman FS, Chaillan FA, Soumireu-Mourat B (1993) Long-term potentiation in rat piriform cortex following discrimination learning. Brain Res 601:265-272.

Saar D, Grossman Y, Barkai E (1998) Reduced after-hyperpolarization in rat piriform cortex pyramidal neurons is associated with increased learning capability during operand -conditioning. Eur J Neurosci 10:1518-1523.

Schoenbaum G, Eichenbaum H (1995a) Information coding in the rodent prefrontal cortex. I. Single-neuron activity in orbitofrontal cortex compared with that in pyriform cortex. J Neurophysiol 74:733-750.

Schoenbaum G, Eichenbaum H (1995b) Information coding in the rodent prefrontal cortex. II. Ensemble activity in orbitofrontal cortex. J Neurophysiol 74:751-762.

Schoenbaum G, Chiba AA, Gallagher M (1999) Neural encoding in orbitofrontal cortex and basolateral amygdala during olfactory discrimination learning. J Neurosci 19:1876-1874.

Staubli U, Fraser D, Farady R, Lynch G (1987) Olfaction and the "data" memory system in rats. Behav Neurosci 101:757-765.

Stripling JS, Patneau DK, Gramlich CA (1988) Selective long-term potentiation in the pyriform cortex. Brain Res 441:281-291.

Stripling JS, Patneau DK, Gramlich CA (1991) Characterization and anatomical distribution of selective long-term potentiation in the olfactory forebrain. Brain Res 542:107-122.

Tseng GF, Haberly LB (1988) Characterization of synaptically mediated fast and slow inhibitory processes in the piriform cortex in an in vitro slice preparation. J Neurophysiol 59:1352-1376.

Wilson MA, McNaughton BL (1994) Reactivation of hippocampal ensemble memories during sleep. Science 265:676-679.

Wu LG, Saggau P (1994) Presynaptic calcium increased during normal synaptic transmission and paired-pulse facilitation, but not long-term potentiation in area CA1 of hippocampus. J Neurosci 14:645-654. 